\title{
User-Centred Design of a Digital Care Plan for Patients and Professionals in Cross- Organisational Teams
}

\author{
Berglind F. SMARADOTTIR ${ }^{\mathrm{a}, \mathrm{b}, 1}$, Gro-Hilde SEVERINSEN ${ }^{\mathrm{a}}$, Aslak STEINSBEKK ${ }^{\mathrm{a}, \mathrm{c}}$ \\ and Gro K.R. BERNTSEN ${ }^{\mathrm{a}, \mathrm{d}}$ \\ ${ }^{a}$ Norwegian Centre for E-health Research, University Hospital of North Norway, \\ Tromsø, Norway \\ ${ }^{\mathrm{b}}$ Department of Information and Communication Technology, University of Agder, \\ Grimstad, Norway \\ ${ }^{\mathrm{c}}$ Department of Public Health and Nursing, Norwegian University of Science and \\ Technology, Trondheim, Norway \\ ${ }^{\mathrm{d}}$ Department of Community Medicine, The Arctic University of Norway, Tromsø, \\ Norway
}

\begin{abstract}
Care for patients with multimorbidity and long-term complex needs is costly and with demographic changes this group is growing. The research project Dignity Care addresses how to improve the care for this patient group by studying how a conceptual shared digital care plan for complex clinical pathways can guide and support cross-organisational care teams. This paper presents the user-centred design process for the digital care plan development. Panels of patients and health care professionals will participate in co-creation user workshops and simulation of complex patients' pathways. The main contribution from this work is recommendations for how to actively involve user groups in digital health development, applying a partly remote approach of user-centred design methodology during the Covid-19 pandemic.
\end{abstract}

Keywords. User-centred Design, Patient-centred Care, Digital Care Plan, Patient Pathways, Information Exchange

\section{Introduction}

The care for multimorbid patients with long-term and complex needs is extraordinarily costly, and with demographic changes in society this group is growing and threatening the sustainability of the health care systems [1][2]. This patient group dominates the $10 \%$ that need $2 / 3$ of the care budget [3][4], and are involved in multiple care processes across care providers, organisations and care levels over long periods [5]. Health care organisations tend to have a silo structured organisation, which together with limitations in legal regulations impedes the digital information flow and collaborative work. However, there are numerous movements to improve this situation, making the need for

\footnotetext{
${ }^{1}$ Corresponding author, Berglind Smaradottir, Norwegian Centre for E-health Research, Post Box 35, N9038 Tromsø, Norway; E-mail: berglind.smaradottir@ehealthresearch.no.
} 
knowledge on what are the effective elements that need to be implemented in digital tools to ensure cooperation and quality of care.

In this context, the research project Dignity Care (2021-2025) addresses research gaps on how to improve the individualised care for multimorbid patients with long-term needs by studying how a conceptual shared digital care plan for complex patient pathways can guide and support multi-disciplinary and cross-organisational health teams [6], in line with national goals by Norwegian health authorities [7]. To do so, user involvement is seen as central and a plan has been made for both ensuring user participation in the project and answering: What are the benefits and constraints of performing a partly remote digital approach in a user-centred design methodology?

This paper presents the user-centred design approach for developing a digital care plan, together with panels of patients and health professionals in the Dignity Care project.

\section{Methodology}

The Dignity Care project employs user-centred design principles for developing the digital care plan [8][9]. The project is in an early study phase, and the main contribution of this paper is the methodological procedure, with complex user-involvement and a partly remote execution because of Covid-19 social distancing restrictions.

This paper reports from the first phase of the project, where eight patient partners, who all represent the target patient group with multi-morbidity and complex long-term needs were recruited to a patient panel through patient organisations and the professional contacts in the authors' network. The patient panel has contributed in digital workshops for detailed planning of how to actively involve users in this project. In the workshops, the patient panel was introduced to the project objectives and aims and familiarised with the research team. They were informed about user-centred design in general and the expected commitment. The members of the patient panel already in the initial dialogue grasped the general concept of the digital tool, and added their own descriptions of functionality that they felt would be essential. They described that there was a need for a "life perspective" rather than an episodic focus. All conditions, and the interaction between them need to be brought to the attention of all professional users, to get a uniform overview of the complex issues. The project aims at recruiting two more patient partners to the panel.

The Dignity Care project involves three health organisation partners, that are located in different health regions of Norway and have been involved in the planning of the project. The project will establish a panel of health care professionals from the partner organisations, building on the same principles as the panel of patients. Two of the organisations were recently part of an explorative field study that targeted mapping and understanding of the existing complex patient pathways and the digital workflow in large organisations, to create a better understanding of the process [10][11]. The study showed lacking interoperability between digital systems and a limited support for teamwork and collaboration both within and across organisations, causing manual efforts to maintain the information flow. Another issue was fragmented overview of long-term trends for complex patients and lacking decision support for clinical interventions [12]. The Dignity Care project aims to contribute with solving those issues on a conceptual level through the work on the digital care plan for complex patient pathways, where the involved health 
team members have access to the same information which includes the goals for the patient, treatment goals and a timeline for tasks that are dedicated to specific resources.

The research team consists of people with expertise in human factors in design, medical informatics, public health and medicine, and at least two team members will be present in sessions together with the panels of patients and professionals. All participation in the study is voluntary and includes a signed individual consent form.

\section{Results}

This section describes the tentative plan for the user-centred design process of the digital care plan development. The patient and health professional panels will contribute in a partially remote co-creation procedure consisting of the following steps: 1) user needs and requirements elicitation in digital workshops, 2) individual semi-structured interviews with members of patient panel targeting the personal care experience with mapping of gaps and critical points to evaluate, 3) construction of 10 anonymised scenarios/use cases, where the experienced gaps in step 2 are present,. 4) drafting of a patient-centred, integrated and proactive care plan together with each individual patient partner and professional care team, involving usual care services so that the plan is realistic and 5) define the needed functionality of the digital care plan.

In addition, the functions of the electronic health record systems in the involved partner organisations will be studied for mapping the communication and information flow, to be used as inspiration for the digital high-fidelity prototype (wireframes) creation. The wireframes will have some implemented active functions and others as visual elements in the graphical user interface, and containing information from the scenarios/use cases.

Members from both panels will participate in user evaluations of the prototype. The evaluation will be executed as a simulation in a usual care setting where all activities are monitored and recorded remotely. A scored evaluation system will be utilised during the simulation, using principles derived from serious gaming. In short, the scenarios/use cases will contain elements based on the critical situations identified, that the professionals are expected to identify and manage according to principles of personcentered, integrated and proactive care. The tools professionals use, should support them in succeeding at these challenges in a better way than the original experience of the patient.

The patient role in the simulation will be played by a professional actor, and the professional roles by healthcare partners in the project. Retrospectively, the panels together with the research team watch the videos in a digital debriefing session to reflect on how they performed, the information flow, the role of the technology and discuss possible improvements. The aim of the user evaluations is to evaluate the performance of the digital care plan prototype in a future prospective-evaluation of the digital care plan, in terms of - What functionality supports the best practice management of complex patient pathways in cross-organisational care delivery?

For data collection purposes, audio-video recording will be made of all interviews, workshops and simulation sessions for retrospective analysis, and a mixed-methods approach will be applied in the data analysis [13][14] as there are both qualitative and quantitative elements. The results from the study execution will be published at a later stage. 


\section{Discussion and Conclusion}

The main contribution of this paper relates to applying user-centred design methodology with a partly remote approach for active end-user contribution in digital health development. Traditionally, in-house meetings are used for data collection through cocreation workshops and project seminars. A digital approach might facilitate an increased user-involvement in the co-creation process, as meetings can be organised with a more frequent schedule and with less inconvenience such as extensive travelling time for the participants and reduced associated costs. This is beneficial in our case, as the project participants are spread over large geographic distances and have multiple diseases that can make travelling challenging and stressful. Less physical interaction minimises the risk of contact spread of Covid-19, which is an important consideration with project participants in elevated risk demographic. However, there are some constraints as well. The digital platform has to be carefully chosen regarding user-friendliness and include an organised technical user support, in case of technical failure for organiser or participants. A digital event has less social interaction and participants might be unfamiliar to each other, which stresses the importance of a clear structure, an actively managed agenda and facilitation of the active voice of each participant. For health and safety reasons, physical comfort breaks are of importance also in digital events.

This paper has some limitations, such as focusing on methodology and not on empirical results. However, the project is in an early phase and the benefit of involving real patients and health professionals in the panels, is their first hand experiences of complex clinical pathways. The panels' participants are included as full project members, and their contribution aims at increasing the quality of care, patient safety, and clinical outcomes for patients with complex pathways. The research team has expertise in the user-centred design domain, and this paper is intended for sharing reflections and knowledge on partly remote procedures when designing digital health developments.

Future work in the Dignity Care project is associated with execution of the full cocreation process together with the project's panels. The outcome is a methodology for testing the effect of the conceptual digital care plan and the care process that results from it, and deployment of the application for an extensive effect study in a Randomised Controlled Trial (RCT). The number of scenarios/use cases $(n=10)$ is related to calculation and validation of data points in the RTC.

\section{Acknowledgement}

The authors thank the members of the patients panel for their disinterested contribution.

\section{References}

[1] Violan C, Foguet-Boreu Q, Flores-Mateo G, et al. Prevalence, determinants and patterns of multimorbidity in primary care: a systematic review of observational studies. PLoS One. 2014 9(7):e102149.

[2] van Oostrom SH, Gijsen R, Stirbu I. et al. Time trends in prevalence of chronic diseases and multimorbidity not only due to aging: data from general practices and health surveys. PLoS One. 2016 11(8):e0160264.

[3] Zulman DM, Pal Chee C, Wagner TH, et al. Multimorbidity and healthcare utilisation among high-cost patients in the US Veterans Affairs Health Care System. BMJ Open. 2015 5(4):e007771. 
[4] Wang L, Si L, Cocker F, Palmer AJ, Sanderson KA. A Systematic Review of Cost-of-Illness Studies of Multimorbidity. Appl Health Econ Health Pol. 2017 16(1):1-15.

[5] Berntsen G, Strisland F. Malm-Nicolaisen K, et al. The evidence base for an ideal care pathway for frail multi-morbid elderly: A combined scoping and systematic intervention review. J Med Internet Res. 2019 21(4):e12517.

[6] Norwegian Centre for E-health Research. Dignity Care. [cited 2021 Jan 30]. Available at: https://ehealthresearch.no/en/projects/dignity-care.

[7] Norwegian Ministry of Health and Care Services. National Health and Hospital Plan 2020-2023. Report No. 7 (2019-2020) to the Storting. Available at: https://www.regjeringen.no/en/dokumenter/meld.-st.-720192020/id2678667/, Accessed 20211 March.

[8] Ergonomics of human system interaction. ISO 9241-210: 2019. Part 210: Standard for human-centred design for interactive systems. International Standardization Organization (ISO), Switzerland.

[9] Gulliksen J, Göransson B, Boivie I, Blomkvist S, Persson J, Cajander Å. Key principles for user-centred systems design. Behav Inf Technol. 2003 22(6):397-409.

[10] Smaradottir BF, Berntsen GKR, Fensli RW. How to enhance digital support for cross-organisational health care teams? A user-based explorative study. J Healthc Eng. 2020:8824882.

[11] Smaradottir BF, Fensli RW. Evaluation of the technology use in an inter-disciplinary patient-centered health care team. Stud Health Techn Inform. 2019 257:388-92.

[12] Smaradottir, BF. A Comparison of the user experiences of primary care electronic health record systems. Adv Intell Syst. 2020 1205: 233-38.

[13] Teddlie C, Tashakkori A. Mixed methods research. Denzin, Norman; Lincoln, Yvonna (eds.), The Sage handbook of qualitative research, SAGE; 2011. p. 285-300.

[14] Johnson RB, Onwuegbuzie AJ, Turner LA. Toward a definition of mixed methods research. J Mix Methods Res. 2007 1(2):112-33. 